\title{
A Comparative Histopathology, Serology and Molecular Study, on Experimental Ocular Toxocariasis by Toxocara cat $i$ in Mongolian Gerbils and Wistar Rats
}

\author{
Mohammad Zibaei, ${ }^{1}$ Seyed Mahmoud Sadjjadi, ${ }^{2,3}$ Mehdi Karamian, ${ }^{4}$ Shoji Uga, \\ Ahmad Oryan, ${ }^{6}$ and Seyed Hamidreza Jahadi-Hosseini ${ }^{7}$ \\ ${ }^{1}$ Department of Parasitology and Mycology, School of Medicine, Alborz University of Medical Sciences, P.O. Box 31485561, Karaj, Iran \\ ${ }^{2}$ Department of Parasitology and Mycology, School of Medicine, Shiraz University of Medical Sciences, P.O. Box 713451735, Shiraz, Iran \\ ${ }^{3}$ Basic Sciences in Infectious Diseases Research Center, School of Medicine, Shiraz University of Medical Sciences, \\ P.O. Box 713451735, Shiraz, Iran \\ ${ }^{4}$ Department of Microbiology, School of Medicine, Birjand University of Medical Sciences, P.O. Box 917751365, Birjand, Iran \\ ${ }^{5}$ Department of Parasitology, Faculty of Health Sciences, Kobe University Graduate School of Health Sciences, \\ P.O. Box 6540142, Kobe, Japan \\ ${ }^{6}$ Department of Pathology, School of Veterinary Medicine, Shiraz University, P.O. Box 713451735, Shiraz, Iran \\ ${ }^{7}$ Department of Ophthalmology, Khalili Hospital, Shiraz University of Medical Sciences, P.O. Box 713451735, Shiraz, Iran
}

Correspondence should be addressed to Seyed Mahmoud Sadjjadi; smsadjjadi@sums.ac.ir

Received 27 April 2013; Revised 22 July 2013; Accepted 29 July 2013

Academic Editor: Christen Rune Stensvold

Copyright ( 2013 Mohammad Zibaei et al. This is an open access article distributed under the Creative Commons Attribution License, which permits unrestricted use, distribution, and reproduction in any medium, provided the original work is properly cited.

\begin{abstract}
The aim of this study was to compare the performance of three in-house diagnostic tests, that is, histopathology, enzyme-linked immunosorbent assay (ELISA), and polymerase chain reaction (PCR), for the diagnosis after experimental infection with Toxocara cati. Twenty Mongolian gerbils and Wistar rats were divided into ten groups $(n=2 /$ group). Toxocara cati infections were established in Mongolian gerbils and Wistar rats by administering doses of 240 and 2500 embryonated Toxocara cati eggs by gavage, respectively. Tissue sections were stained with Haematoxylin and Eosin and observed under the light microscope. Sera and vitreous fluid collected from separate infected groups were tested against Toxocara cati antigens, for 92 days postinfection. Genomic DNA was extracted from formalin-fixed paraffin-embedded (FFPE) blocks, and aqueous fluids belong to the animals. The histopathology test gave negative results among the groups of animals examined between 5 and 92 days postinfection. The ELISA results showed that anti-Toxocara antibodies have risen between 7 and 61 days postinfection in sera and vitreous fluid in the animals infected, respectively. Analysis of PCR products revealed positive band ( $660 \mathrm{bp})$ in the orbital tissue infected Mongolian gerbils at 5 days postinfection. Of the three evaluated methods, the PCR could be recommended for scientific and laboratory diagnoses of toxocariasis in experimentally infected animals.
\end{abstract}

\section{Introduction}

Toxocariasis is one of the most commonly reported zoonotic helminthic infections in the world. Both Toxocara canis (a round worm of dogs) and Toxocara cati (common round worm of cats) cause toxocariasis. Ingestion of Toxocara species eggs containing third-stage larvae, due to geophagia, pica, and the consumption of contaminated raw meat or liver, is considered as possible cause of visceral larva migrans (VLM) or ocular larva migrans (OLM) $[1,2]$.

Ocular infection with Toxocara larvae species is uncommon but of interest to ophthalmologists because it largely affects the young people in whom it can cause significant ocular morbidity or even blindness. The clinical sign of ocular toxocariasis in human often includes diminished vision, leukocoria, red eye, and strabismus. The diagnosis of 
suspected ocular toxocariasis is supported by the presence of chorioretinal or focal lesions in posterior eye segment in the presence of positive serology $[3,4]$. Toxocara cati (T. cati) is the common worm of cats and its third-stage larvae are possible cause of OLM. Several studies have been reported that human can be infected by the ingestion of $T$. cati embryonated eggs with contaminated soil or eating larvae within paratenic hosts including birds and small rodents. There is little finding on the location of $T$. cat $i$ larvae in paratenic hosts including the eye as causative agents of human toxocariasis $[5,6]$.

In the last few years, several authors have approached diagnosis through the specific detection of antigens or antibodies $[7,8]$. Nevertheless, the histopathological demonstration of larvae is relatively intensive, especially due to low larvae burden $[9,10]$. Thus, the development of new and more sensitive diagnostic tools is needed. Desirably, theis new approach should permit the diagnosis in early infection, to prevent the development of severe pathologies associated with later infection. Application of molecular methods for the diagnosis of Toxocara infections has been increasing. Several studies have reported the usefulness of polymerase chain reaction (PCR) for the characterization and the diagnosis of toxocariasis compared to other parasitic diseases in natural or experimentally infected animals $[11,12]$.

Based on the widespread use of polymerase chain reaction for the diagnosis of parasitic diseases, and to the best of our knowledge that there are no reports about of use of PCR in diagnosis of ocular toxocariasis, this study was designed to evaluate PCR for being used in the diagnosis after experimental ocular infection with $T$. cat $i$ third-stage larvae, compared to histopathology and Enzyme-Linked Immunosorbent Assay (ELISA).

\section{Materials and Methods}

2.1. Animals and Experimental Infections. Ten Mongolian gerbils (one-month-old males) and ten Wistar rats (onemonth-old males) were assigned in ten experimental groups ( $n=2$ /group): each two animals of the same, were infected orally with approximately 240 and 2500 embryonated $T$. cat $i$ eggs, respectively $[6,13-15]$. The eggs were derived from adult female worms and were embryonated according to our previous paper [16]. The animals were maintained under standard conditions, in an environment with controlled temperature and humidity. The inoculated animals were euthanized on days 5, 30, 49, 70, and 92 postinfection (PI). The vitreous fluid which is a jelly compound was taken from the eyeballs of infected animals, using a 22-gauge needle linked to a powerful syringe and stored at $-30^{\circ} \mathrm{C}$ until used. The experimental protocol was approved by the Ethical Committee of the Shiraz University of Medical Sciences.

2.2. Preparation of T. cati Excretory-Secretory (ES) Antigens. ES antigens for ELISA assay were obtained from thirdstage larvae $\left(\mathrm{ES} / \mathrm{L}_{3}\right)$. Adult worms of $T$. cat $i$ were collected from the intestines of infected stray cats and the eggs were isolated from uteri of female worms, were decoated by sodium hypochlorite $(7 \% \mathrm{w} / \mathrm{v})$, and were embryonated in $2.5 \%$ formalin/ringer solution while being incubated at an atmosphere of $5 \% \mathrm{CO}_{2}$ in $25^{\circ} \mathrm{C}$ for 3 weeks [16]. The larvae were collected aseptically from a Baermanns apparatus. ES antigens of $T$. cati were prepared from third-stage larvae by the method of de Savigny (1975) with modification [17].

2.3. Pathological Study. Samples of eyeball from the infected animals were fixed in $10 \%$ neutral buffered formalin, dehydrated with graded ethanol, and embedded in paraffin. Tissue sections of $5 \mu \mathrm{m}$ thickness were stained with Haematoxylin and Eosin (H \& E) and after mounting, were observed under the light microscope.

2.4. Immunological Study. ELISA was carried out in flatbottom96 microplates. The ES antigen was collected from culture supernatant of $T$. cati third-stage larvae as described. The plates were coated with $100 \mu \mathrm{L}$ of ES antigen in coating buffer $(0.05 \mathrm{M}$ carbonate bicarbonate-buffer, $\mathrm{pH}$ 9.6) and incubated at $4^{\circ} \mathrm{C}$ overnight. Excess antigen was removed by washing the plate three times in phosphate buffered salineTween 20 (PBST, pH 7.4 containing 0.05\% Tween 20). Excess binding sites were blocked for $1 \mathrm{hr}$ at $37^{\circ} \mathrm{C}$ with $100 \mu \mathrm{L}$ of bovine serum albumin (1\% dilution in PBS). The wells were washed, and then $100 \mu \mathrm{L}$ of diluted serum (1:100 in PBS) and vitreous humor (1:2 in PBS) were added to each well and incubated for $1 \mathrm{hr}$ at $37^{\circ} \mathrm{C}$. The plates were washed as before, and $100 \mu \mathrm{L}$ of horseradish peroxidase-conjugated sheep anti-mouse IgG at a 1/1000 dilution in PBST was added and incubated for one hour at $37^{\circ} \mathrm{C}$. After washing, the plates were incubated with chromogen/substrate $(100 \mu \mathrm{L} /$ well ABTS peroxidase substrate) and the reaction was terminated with peroxidase stop solution $(50 \mu \mathrm{L}$, diluted $1: 5)$ after 5 min. The absorbance at $405 \mathrm{~nm}$ was monitored with a MUNK microplate reader. The cutoff point was set as $2 \mathrm{SD}$ above the mean of control samples.

2.5. DNA Isolation and PCR Analyses. Extraction of DNA was done from formalin-fixed paraffin-embedded (FFPE) blocks, and aqueous fluids belong to gerbils and rats. FFPE blocks were cut and deparaffinized using Xylol due to standard protocols. Genomic DNA was isolated from individual samples using QIAamp DNA Mini and Blood Mini Handbook Kit (Qiagen, Hilden, Germany) according to the manufacturer's instruction. The specific forward primer JW4 $\left(5^{\prime}\right.$-ACTGTCGAGGATGAGCGTGA-3') [18] was used with NC2 primer (reverse: $5^{\prime}$-TTAGTTTCTTTTCCTCCGCT-3') [11] to amplify partial ITS-1, complete $5.8 \mathrm{~S}$, and ITS-2 rDNA of T. cati. PCR reaction was performed in $10 \mathrm{mM}$ Tris-HCL, $\mathrm{pH} 8.8,1.5 \mathrm{mM} \mathrm{MgCl}_{2}, 50 \mathrm{mM} \mathrm{KCl}, 0.1 \%$ Triton X-100, dNTP $200 \mu \mathrm{M}$, primers $1 \mu \mathrm{M}$, and $1 \mathrm{U}$ Taq polymerase per $25 \mu \mathrm{L}$. The reaction was carried out under the following conditions: $94^{\circ} \mathrm{C}$ for $45 \mathrm{~s}, 58^{\circ} \mathrm{C}$ for $45 \mathrm{~s}, 72^{\circ} \mathrm{C}$ for $90 \mathrm{~s}$, and final extension $72^{\circ} \mathrm{C}$ for $10 \mathrm{~min}$. Corresponding PCR products were electrophoresed in $1.4 \%$ agarose gel with $0.5 \mu \mathrm{g} / \mathrm{mL}$ ethidium bromide, and a 100-bp ladder was used as DNA sizemarker for estimating the size of the amplicons and photographed using a gel documentation system (UV Transilluminator). 


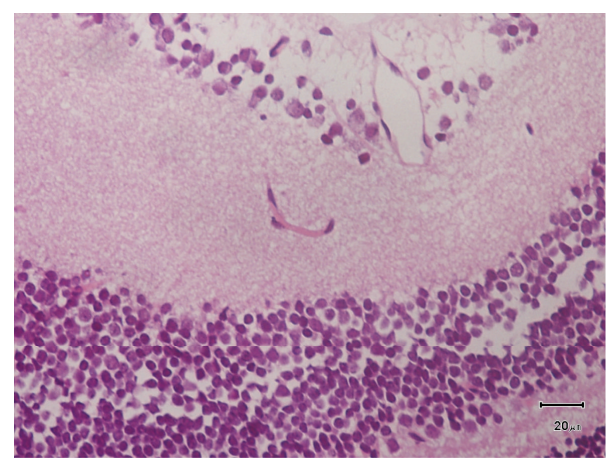

FIGURE 1: The negative reaction in the retina of the infected animals (paraffin section stained, $\mathrm{H}$ with $\mathrm{E}$ ).

\section{Results}

In an attempt to identify the optimal procedure for the diagnosis of ocular Toxocara infection, the performance and agreement among the three different methods, namely, histopathology, ELISA, and PCR methods, were evaluated.

3.1. Pathological Observation. Figure 1 corresponds to image of the ocular structure of an infected animal. In none of the studied animals, we observed peripheral granuloma in the posterior pole or retrolental hyaline membranes.

3.2. ELISA for Anti-Toxocara Antibodies. The ELISA results were positive in sera and aqueous fluid for Mongolian gerbils and Wistar rats examined at 1 and 8 weeks PI, respectively. Specimens with an OD (optical density) reading greater than 0.300 and less than 0.260 can be interpreted with assurance as positive and negative, respectively. Caution may be necessary with specimens with inter with mediate values. At the seventh day PI, the test was positive in 15 out of 20 animals (75\% sensitivity), where the animals showed antiToxocara antibodies in sera. The test was positive at an OD greater than 0.310 and higher in 10 Mongolian gerbils and 5 Wistar rats. At the sixty-one day PI the test was positive for aqueous fluid in 11 out of 20 animals (55\% sensitivity), where the test was positive at an OD of 0.300 or more in nine Mongolian gerbils and two Wistar rats (Figure 2).

3.3. PCR Analysis. Analysis of PCR products revealed positive band $(660 \mathrm{bp})$ in the orbital tissue infected Mongolian gerbils at 5 days PI. The DNA of T. cati was found in the vitreous fluid of experimentally infected Mongolian gerbils. No positive band was shown in samples of Wistar rats (Figure 3).

\section{Discussion}

Toxocariasis is an important infection among socioeconomically disadvantaged young children and is low in adults. Ocular larva migrans occurs when Toxocara larvae reach eye tissue, where they induce inflammatory reaction, frequently without the sign or symptoms that accompany the visceral toxocariasis [19-22]. The diagnosis of ocular larva migrans is

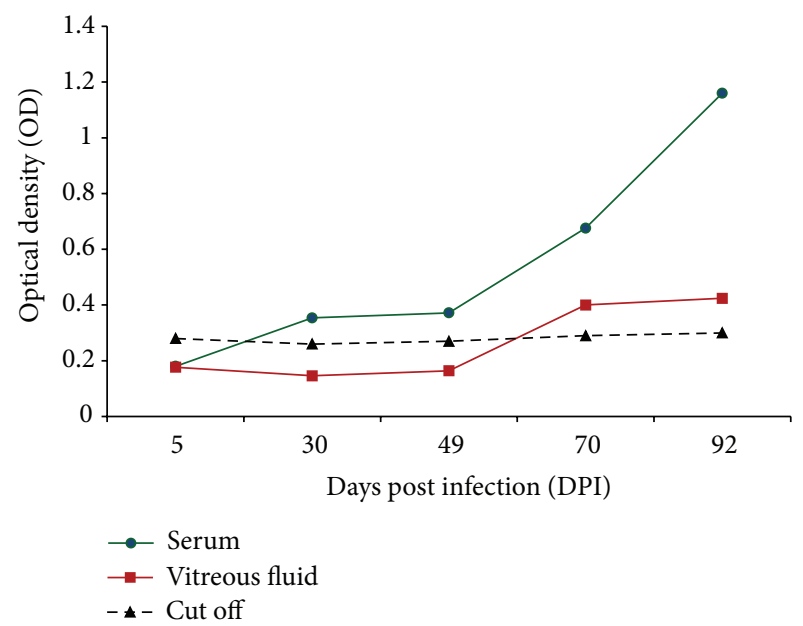

FIgURE 2: Development of specific Toxocara cati L2S ES antigens in infected Mongolian gerbils and Wistar rats with 240 and 2500 T. cati embryonated eggs, respectively. OD values of uninfected animals were lower than the negative cutoff 0.260 , and those of infected animals were higher than positive cutoff 0.300 .

based on retinoscopic and retinographic studies, and is confirmed by the finding of larvae in lesions [23]. The presence of anti-Toxocara antibodies or antigens in vitreous humor has been reported to be a sensitive method for detecting toxocariasis in infections or human ocular toxocariasis [25].

In this study we compared the performance of three different diagnostic techniques including: histopathology, indirect ELISA and PCR on eyeball and retina tissue during the infection of Mongolian gerbils and Wistar rats from 5 to 92 days PI.

The infection with $T$. cati in the animals not caused important histological alternative in cellular structures of the eye. Histopathologic examination did not detect foreign bodies or tumors in the affected region, and the ophthalmic examination did not suggest an infectious process. Serological testing is the primary diagnostic tool in patient suspected for ocular and visceral larva migrans, but these tests are insufficient, as cross-reactions may occuring. For example, crossreactivity has been reported between T. canis and Trichinella spiralis, and T. canis and Ascaris lumbricoides [26]. Sommerfelt et al. (2006) detected specific antibodies against T. canis with ELISA, in eighteen pigs at 7 days PI, with high titers persisting over an 84-day period [27]. In current study, an immunological response in sera was early, 7 days PI, and was maintained throughout the study (92 days) and these results are similar to those of other authors. Beside, from 61 days of the infection antibodies in vitreous fluid were detected.

An alternative approach to detection of toxocariasis is to use a molecular-based-method such as PCR. When PCR was applied to eye tissue and aqueous fluid of infected Mongolian gerbils, detection of the specific amplification started at 5 days PI. This was not possible in other stage of infection (30, 49, 70 and 92 days PI). Our PCR approach in retina demonstrated the presence of the parasite genome before one week PI, while the histological method was not useful for detection of parasite at the retinal and subretinal levels. 


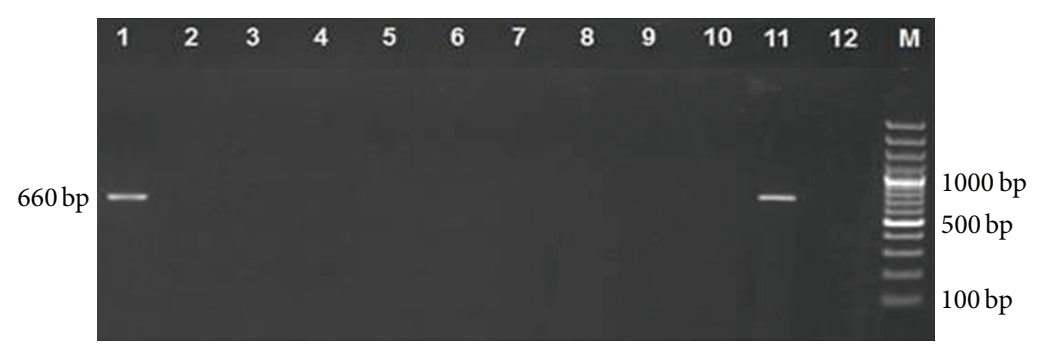

(a)

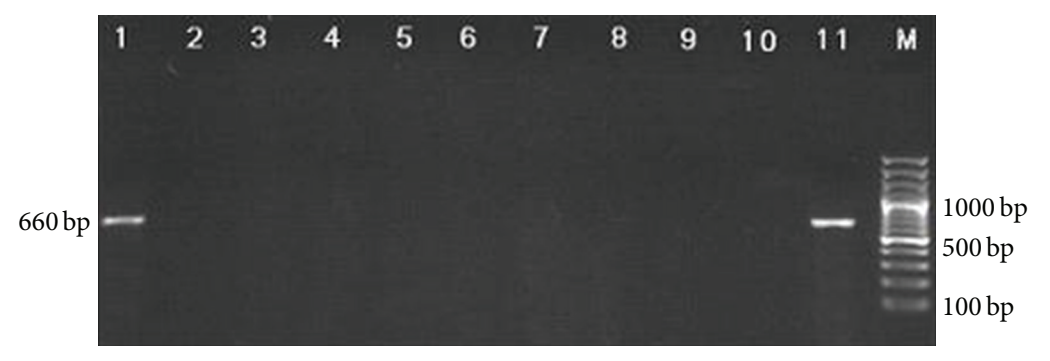

(b)

Figure 3: (a) Analysis of PCR products (eye tissue of infected animals) by 1.4\% agarose gel electrophoresis. Lanes 1-5: days 5 to 92 PI of Mongolian gerbils; lanes 6-10: days 5 to 92 PI of Wistar rats; lane 11: DNA of Toxocara cati adult worm as positive control; lane 12: negative control; lane M: 100-1500 bp DNA size marker. (b) DNA amplification of vitreous fluid samples of infected animals by PCR on 1.4\% agarose gel. Lanes 1-5: days 5 to 92 PI of Mongolian gerbils; lanes 6-10: days 5 to 92 PI of Wistar rats; lane 11: DNA of Toxocara cati adult worm as positive control; lane M: 100-1500 bp DNA size marker.

The PCR in vitreous humor could also be more sensitive than the detection of antibodies by ELISA in the animals in early infection, since prominent positive serology was detectable from 61 days PI. This period in animal challenge, although not strictly equivalent to human infection in clinical course, could be considered as an acute infection in patients regarding the parasitic migration and development. It seems that the transmission of larvae was first from intestine to the liver, lung and eye, and then 5 days PI they migrated to other organs. Sometimes larvae return back to the eye to create lesions at the end of migration. It has been demonstrated that Toxocara larvae may migrate and persist in the eye of experimental infected animals. This opinion is challenged by several authors, who argued that Toxocara is as a causative ocular toxocariasis $[28,29]$. In a relatively similar study, Cho et al., (2007) infected Mongolian gerbils with 1000 Toxocara ova and reported that $10 \%$ of infective larvae were found before day 5 in liver tissue and also showed that ophthalmoscopically, a motile larva was observed in the retina at 14 days PI [30]. In earlier studies, investigators revealed the parasite DNA by PCR during early infection periods in the animals model for Trypanosoma cruzi and Toxoplasma gondii, thus permitting the diagnosis of infection earlier than when conventional diagnostic techniques such as microscopy or serology are being used $[31,32]$.

\section{Conclusion}

Histopathology, serology (ELISA), and molecular (PCR) methods tested for diagnosis of ocular toxocariasis caused by $T$. cat $i$ in experimentally infected Mongolian gerbils and Wistar rats revealed that molecular method was the most sensitive and superior compared to both histopathology and ELISA in the early diagnosis of ocular infection. The PCR provided a rapid, reliable and sensitive means by which one establish the exact etiology of such parasitic infection so that optimal therapy can be started promptly. The superior performance of the PCR in detecting infected Mongolian gerbils and Wistar rats in this small experimental population warrants further evaluation in a larger population of animal models and humans naturally infected with $T$. cati.

\section{Conflict of Interests}

The authors report no conflict of interest in this work.

\section{Acknowledgments}

The technical assistance of Mrs. S. Kazemian and Mr. B. Farhang Mehr of the Department of Parasitology and Mycology, School of Medicine, Shiraz University of Medical Sciences Iran is acknowledged with pleasure. The critical comments of Professor Akao from Section of Environmental Parasitology, Graduate School of Tokyo Medical and Dental University, Japan, are gratefully acknowledged. This study was funded by Shiraz University of Medical Sciences, Shiraz, Iran, Grant no. 4108.

\section{References}

[1] S. Azizi, A. Oryan, S. M. Sadjjadi, and M. Zibaei, "Histopathologic changes and larval recovery of Toxocara cat i in experimentally infected chickens," Parasitology Research, vol. 102, no. 1, pp. 47-52, 2007. 
[2] D. Despommier, "Toxocariasis: clinical aspects, epidemiology, medical ecology, and molecular aspects," Clinical Microbiology Reviews, vol. 16, no. 2, pp. 265-272, 2003.

[3] R. S. Clemett, H. J. Williamson, R. R. Hidajat, R. A. Allardyce, and A. C. Stewart, "Ocular Toxocara canis infections: diagnosis by enzyme immunoassay," Australian and New Zealand Journal of Ophthalmology, vol. 15, no. 2, pp. 145-150, 1987.

[4] L. de Visser, A. Rothova, J. H. de Boer et al., "Diagnosis of ocular toxocariasis by establishing intraocular antibody production," American Journal of Ophthalmology, vol. 145, no. 2, pp. 369-374, 2008.

[5] N. Akao and N. Ohta, "Toxocariasis in Japan," Parasitology International, vol. 56, no. 2, pp. 87-93, 2007.

[6] M. Zibaei, S. M. Sadjjadi, and S. Uga, "Experimental Toxocara cati infection in gerbils and rats," Korean Journal of Parasitology, vol. 48, no. 4, pp. 331-333, 2010.

[7] T. H. Dzbenski, W. Hautz, and E. Bitkowska, "Experimental toxocariasis in rabbits: immunological markers of ocular infections," Wiadomości Parazytologiczne, vol. 47, no. 4, pp. 591-596, 2001.

[8] R. Sakai, H. Kawashima, H. Shibui, K. Kamata, C. Kambara, and H. Matsuoka, "Toxocara cati-induced ocular toxocariasis," Archives of Ophthalmology, vol. 116, no. 12, pp. 1686-1687, 1998.

[9] N. Cardillo, A. Rosa, M. Ribicich, C. López, and I. Sommerfelt, "Experimental infection with Toxocara cati in BALB-c mice, migratory behaviour and pathological changes," Zoonoses and Public Health, vol. 56, no. 4, pp. 198-205, 2009.

[10] K. Taira, Y. Saitoh, and C. M. O. Kapel, “Toxocara cati larvae persist and retain high infectivity in muscles of experimentally infected chickens," Veterinary Parasitology, vol. 180, no. 3-4, pp. 287-291, 2011.

[11] X. Q. Zhu, S. D’Amelio, H. W. Palm, L. Paggi, M. GeorgeNascimento, and R. B. Gasser, "SSCP-based identification of members within the Pseudoterranova decipiens complex (Nematoda: Ascaridoidea: Anisakidae) using genetic markers in the internal transcribed spacers of ribosomal DNA," Parasitology, vol. 124, no. 6, pp. 615-623, 2002.

[12] A. Borecka, J. Gawor, M. Niedworok, and B. Sordyl, "Detection of Toxocara canis larvae by PCR in the liver of experimentally infected Mongolian gerbils (Meriones unguiculatus)," Helminthologia, vol. 45, no. 3, pp. 147-149, 2008.

[13] N. Akao, T. H. Takayanagi, R. Suzuki, S. Tsukidate, and K. Fujita, "Ocular larva migrans caused by Toxocara cati in Mongolian gerbils and a comparison of ophthalmologic findings with those produced by T. canis," Journal of Parasitology, vol. 86, no. 5, pp. 1133-1135, 2000.

[14] E. Hayashi, N. Akao, and K. Fujita, "Evidence for the involvement of the optic nerve as a migration route for larvae in ocular toxocariasis of Mongolian gerbils," Journal of Helminthology, vol. 77, no. 4, pp. 311-315, 2003.

[15] S. Z. Lescano, M. L. Queiroz, and P. P. Chieffi, "Larval recovery of Toxocara canis in organs and tissues of experimentally infected Rattus norvegicus," Memorias do Instituto Oswaldo Cruz, vol. 99, no. 6, pp. 627-628, 2004.

[16] M. Zibaei, S. M. Sadjjadi, S. H. Jahadi Hosseini, and B. Sarkari, "A method for accelerating the maturation of Toxocara cati eggs," Iranian Journal of Parasitology, vol. 2, no. 1, pp. 39-42, 2007.

[17] D. H. De Savigny, "In vitro maintenance of Toxocara canis larvae and a simple method for the production of Toxocara ES antigen for use in serodiagnostic tests for visceral larva migrans," Journal of Parasitology, vol. 61, no. 4, pp. 781-782, 1975.
[18] M. W. Li, R. Q. Lin, H. H. Chen, R. A. Sani, H. Q. Song, and X. Q. Zhu, "PCR tools for the verification of the specific identity of ascaridoid nematodes from dogs and cats," Molecular and Cellular Probes, vol. 21, no. 5-6, pp. 349-354, 2007.

[19] R. Molk, "Ocular toxocariasis: a review of the literature," Annals of Ophthalmology, vol. 15, no. 3, pp. 216-231, 1983.

[20] J. A. Shields, "Ocular toxocariasis. A review," Survey of Ophthalmology, vol. 28, no. 5, pp. 361-381, 1984.

[21] S. H. Gillespie, W. J. Dinning, A. Voller, and N. S. Crowcroft, "The spectrum of ocular toxocariasis," Eye, vol. 7, no. 3, pp. 415418, 1993.

[22] B. Good, C. V. Holland, M. R. H. Taylor, J. Larragy, P. Moriarty, and M. O'Regan, "Ocular toxocariasis in schoolchildren," Clinical Infectious Diseases, vol. 39, no. 2, pp. 173-178, 2004.

[23] L. I. D. C. Zanandréa, G. M. Oliveira, A. S. Abreu, and F. E. L. Pereira, "Ocular lesions in gerbils (Meriones unguiculatus) infected with low larval burden of Toxocara canis: observations using indirect binocular ophthalmoscopy," Revista da Sociedade Brasileira de Medicina Tropical, vol. 41, no. 6, pp. 570-574, 2008.

[24] J.-F. Magnaval, L. Malard, B. Morassin, and R. Fabre, "Immunodiagnosis of ocular toxocariasis using Western-blot for the detection of specific anti-Toxocara IgG and CAPU for the measurement of specific anti-Toxocara IgE," Journal of Helminthology, vol. 76, no. 4, pp. 335-339, 2002.

[25] E. Bertelmann, K.-H. Velhagen, U. Pleyer, and C. Hartmann, "Ocular toxocariasis. Diagnostic and therapeutic options," Ophthalmologe, vol. 100, no. 11, pp. 950-954, 2003.

[26] H. Żarnowska and M. Jastrzębska, "Excretory-secretory larval antigens of Toxocara canis: physico-chemical characteristics and specificity assayed by Western blot technique," Acta Parasitologica, vol. 39, no. 3, pp. 41-45, 1994.

[27] I. E. Sommerfelt, G. Santillán, C. Lopez, M. Ribicich, and A. J. Franco, "Immunological and hematological response in experimental Toxocara canis-infected pigs," Veterinary Parasitology, vol. 96, no. 2, pp. 127-134, 2001.

[28] T. H. Takayanagi, N. Akao, R. Suzuki, M. Tomoda, S. Tsukidate, and K. Fujita, "New animal model for human ocular toxocariasis: ophthalmoscopic observation," British Journal of Ophthalmology, vol. 83, no. 8, pp. 967-972, 1999.

[29] S. V. Santos, S. Z. Lescano, J. M. Castro, and P. P. Chieffi, "Larval recovery of Toxocara cati in experimentally infected Rattus norvegicus and analysis of the rat as potential reservoir for this ascarid," Memorias do Instituto Oswaldo Cruz, vol. 104, no. 6, pp. 933-934, 2009.

[30] S. Cho, M. Egami, H. Ohnuki et al., "Migration behaviour and pathogenesis of five ascarid nematode species in the Mongolian gerbil Meriones unguiculatus," Journal of Helminthology, vol. 81, no. 1, pp. 43-47, 2007.

[31] L. M. Weiss, S. A. Udem, M. Salgo, H. B. Tanowitz, and M. Wittner, "Sensitive and specific detection of toxoplasma DNA in an experimental murine model: use of Toxoplasma gondiispecific cDNA and the polymerase chain reaction," Journal of Infectious Diseases, vol. 163, no. 1, pp. 180-186, 1991.

[32] L. V. Kirchhoff, J. R. Votava, D. E. Ochs, and D. R. Moser, "Comparison of PCR and microscopic methods for detecting Trypanosoma cruzi," Journal of Clinical Microbiology, vol. 34, no. 5, pp. 1171-1175, 1996. 

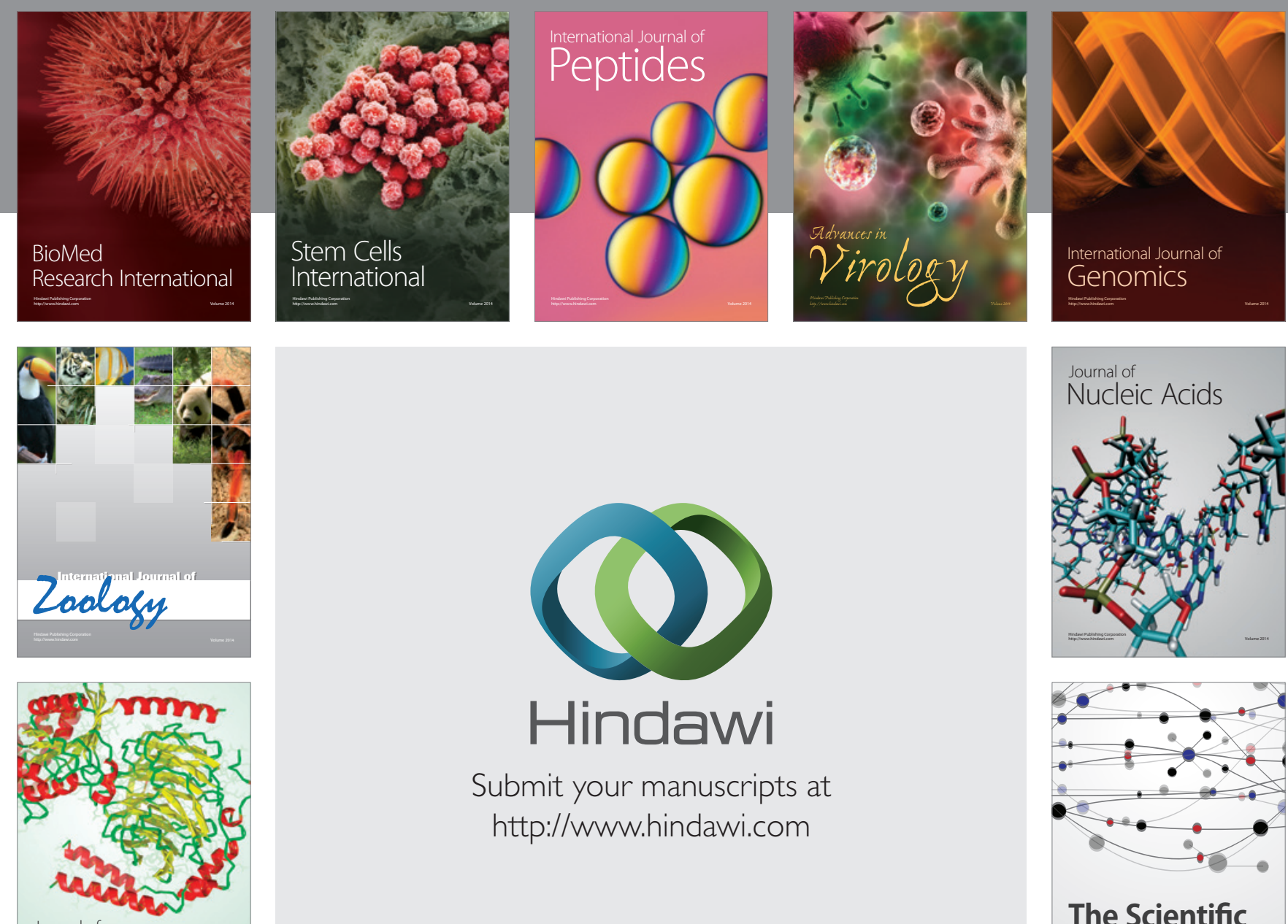

Submit your manuscripts at

http://www.hindawi.com

Journal of
Signal Transduction
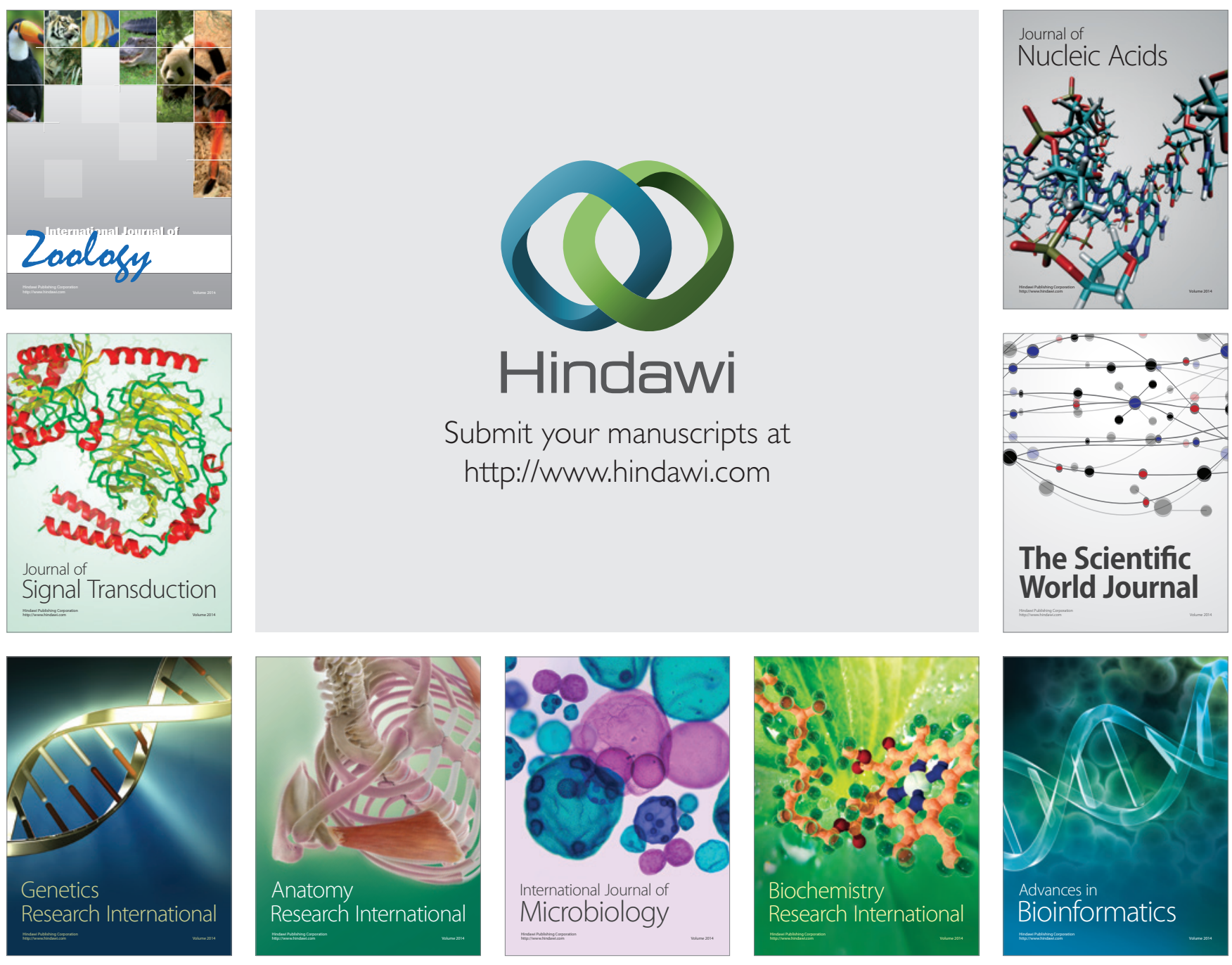

The Scientific World Journal
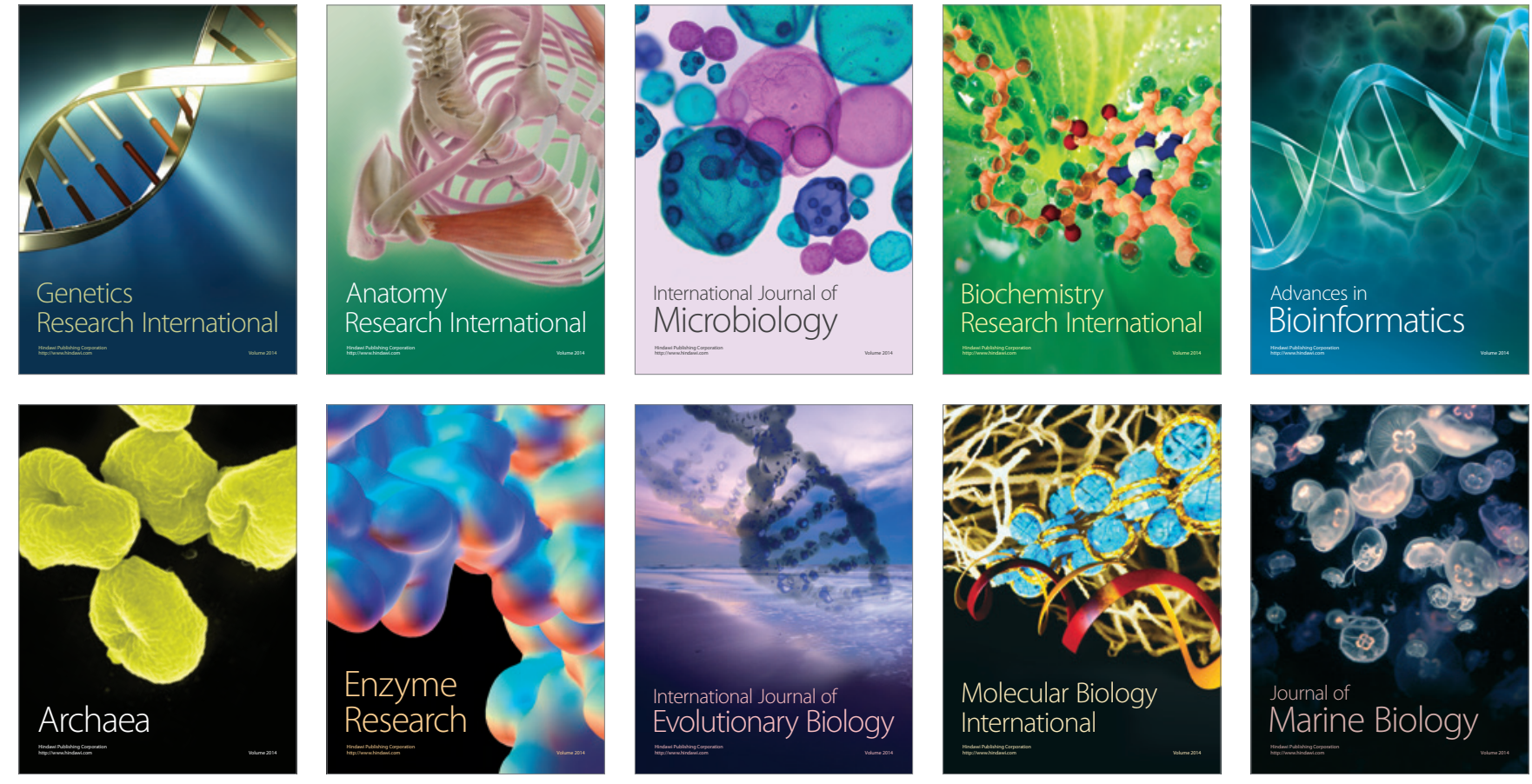